\title{
Circulating Endothelial Progenitor Cells in Crohn's Disease: An EPiC in the Making?
}

\author{
Catharine Dietrich $^{1} \cdot$ Shree Ram Singh ${ }^{1}$
}

Published online: 11 January 2017

(C) Springer Science+Business Media New York (Outside the USA) 2017

The inflammatory bowel diseases (IBDs), divided into Crohn's disease (CD) and ulcerative colitis (UC), are characterized by chronic inflammation of the entire gastrointestinal tract (CD) or colon (UC) associated with unrestrained immune response with activation of CD4+ T cells, expansion of the intestinal microvasculature, and endothelial impairment [1, 2]. Approximately 565,000 residents of the USA are diagnosed with $C D$ each year. Although the exact causes of $C D$ remain unclear, it has been suggested that autoimmune components, immune deficiency, genetic susceptibility, dietary factors, bacteria, and fungi may in part contribute to disease pathogenesis [3-5]. Since current conventional therapies available for $\mathrm{CD}$ only control the inflammatory process in order to avoid structural damage to the intestine, new strategies targeted at underlying disease etiology are needed in order to repair damaged intestinal tissue and the immune system.

In recent years, advances in the understanding of the cell populations involved in pathogenic processes and of the regenerative and immunomodulatory potential of stem cells have opened up a new direction in IBD therapy. In this context, hematopoietic stem cells (HSCs), mesenchymal stem cells (MSCs), endothelial progenitor cells (EPCs), resident stem cells of intestine, and induced pluripotent stem cells (iPSCs) could be helpful in IBD therapy. Studies in the last several years suggest that endothelial impairment substantially contributes to the onset and progression of and recovery from inflammatory

Shree Ram Singh

singhshr@mail.nih.gov

1 Stem Cell Regulation and Animal Aging Section, Basic Research Laboratory, National Cancer Institute, NIH, Frederick, MD 21702, USA conditions [1]. EPCs, derived from bone marrow hematopoietic stem cells, (HSCs) have the capability to migrate to the site of endothelial damage through the peripheral circulation and have been used to treat several inflammatory diseases, ischemic limb disease, peripheral vascular disease, and coronary heart disease [6-8].

Despite these successes, the effectiveness of circulating EPCs in the amelioration of CD remains speculative [1, 9, 10]. Garolla et al. [1] reported a lower number of circulating EPCs in CD patients compared to controls, demonstrating that apoptosis is the principal pathway for EPC reduction. Contrary to the previous study, Masuda et al. [9] reported no significant reduction in the number of circulating EPCs among CD patients as compared to controls. Furthermore, Marlicz et al. [10] described significantly elevated numbers of circulating EPCs in CD patients. Therefore, it is essential to clarify the importance of circulating EPCs toward CD pathophysiology by performing careful studies.

In this issue of Digestive Diseases and Sciences, Boltin et al., in an effort to resolve the issue related to the number of circulating EPCs in context of CD [11], studied $32 \mathrm{CD}$ patients and 51 healthy controls, recruiting $\mathrm{CD}$ patients of varying ancestry, assessing disease activity using the Crohn's disease activity index (CDAI). Among these patients, twenty-eight were being treated with biological therapy (infliximab) and twenty-one were receiving immunomodulators. Boltin et al. [11] isolated the peripheral blood mononuclear cells and evaluated the EPC numbers by fluorescent cell sorting (FACS) analysis employing antibodies to the biomarkers cluster of differentiation (CD)34, vascular endothelial growth factor (VEGF) receptor-2, CD133, and CD45. They reported a significant increase in the percentage of EPCs of the peripheral white blood cells in CD patients as compared to 
healthy controls. Nevertheless, they could not significantly correlate EPC percentages and other factors such as age, gender, CDAI, disease duration, duration of biological therapy, or smoking.

Boltin et al.'s [11] findings mark an important step toward addressing the current controversies in $\mathrm{CD}$ research. Their results are consistent with accumulating evidence that EPCs do in fact participate in the regeneration of damaged epithelium. Actually, in a recent EPC transplantation study using fetal sheep, Wood et al. [12] demonstrated that EPCs can migrate to the intestinal mucosa and participate in vasculogenesis. Given this observation, why were circulating EPC numbers in CD patients reported to be unchanged or even reduced in previous studies? Boltin et al. [11] reasoned that in their study, a high percentage of patients were receiving biological therapy that in turn could assist in recruiting EPCs to the endothelial repair site. In fact, Galiano et al. [13] reported that a local elevated level of VEGF and SDF-1 in ischemic foci may help in EPC recruitment from the circulation to the site of injury to assist diabetic wound healing. Therefore, it is highly likely that an increase in peripheral EPCs may be a sign of treatment feedback, though the authors could not support this association in their study [11]. The present study [11] presents a major advantage over previous studies in terms of clinical variables and number of patients. Nevertheless, there are also several limitations suggested by authors [11] including lack of endoscopic and laboratory measures of disease activity, measuring EPC abundance in tissues, and lack of clinical and demographic associations.

In this article, authors only presented data on the circulating numbers of EPCs in CD patients using flow cytometry; therefore, in future experiments employing large sample of patients, histologic sections by immunohistochemistry to show increased numbers of EPC in and around the gut lesions, especially near newly formed blood vessels, are needed. Exclusive EPC markers will be needed to distinguish EPCs from native endothelial cells. Proliferative ability, survival, and migration efficiency of early and mature EPCs should be tested in order to measure their functionality. Furthermore, evaluation of the mobilization and recruitment of EPCs using CD tissue microarrays or RNA-Seq would be needed in order to unravel novel regulators. Once this new information is available, knowledge of the mechanisms by which EPCs can mobilize and repair endothelial damage in response to chronic inflammation in $\mathrm{CD}$ patients, and the signaling pathways involved in the regulation of endothelial repair should increase. From a therapeutic standpoint, trials of EPC transplantation in $\mathrm{CD}$ patients could help ascertain if this intervention enhances the regeneration process of intestinal tissues.
Enhanced understanding of the etiology of $\mathrm{CD}$ and the mechanisms of endothelial damage and repair will provide useful insights into the $\mathrm{CD}$ pathogenesis, which in turn will inform the design of novel therapeutic strategies in its treatment.

\section{Compliance with ethical standards}

Conflict of interest The authors declare that they have no conflict of interest.

\section{References}

1. Garolla A, D'Incaà $\mathrm{R}$, Checchin $\mathrm{D}$, et al. Reduced endothelial progenitor cell number and function in inflammatory bowel disease: a possible link to the pathogenesis. Am J Gastroenterol. 2009;104:2500-2507.

2. Singh UP, Singh NP, Singh B, et al. Stem cells as potential therapeutic targets for inflammatory bowel disease. Front Biosci (Sch Ed). 2010;2:993-1008.

3. Xavier RJ, Podolsky DK. Unravelling the pathogenesis of inflammatory bowel disease. Nature. 2007;448:427-434.

4. Barrett JC, Hansoul S, Nicolae DL, et al. Genome-wide association defines more than 30 distinct susceptibility loci for Crohn's disease. Nat Genet. 2008;40:955-962.

5. Hoarau G, Mukherjee PK, Gower-Rousseau C, et al. Bacteriome and mycobiome interactions underscore microbial dysbiosis in familial Crohn's disease. MBio. 2016;7:e01250-16.

6. Závada J, Kideryová L, Pytlík R, Vaňková R, Tesař V. Circulating endothelial progenitor cells in patients with ANCA-associated vasculitis. Kidney Blood Press Res. 2008;31:247-254.

7. Povsic TJ, Junge C, Nada A, et al. A phase 3, randomized, double-blinded, active-controlled, unblinded standard of care study assessing the efficacy and safety of intramyocardial autologous CD34+ cell administration in patients with refractory angina: design of the RENEW study. Am Heart J. 2013;165:e2e861.

8. Losordo DW, Kibbe MR, Mendelsohn F, et al. A randomized, controlled pilot study of autologous CD34+ cell therapy for critical limb ischemia. Circ Cardiovasc Interv. 2012;5:821-830.

9. Masuda J, Mitsuyama K, Yamasaki H, et al. Depletion of endothelial progenitor cells in the peripheral blood of patients with ulcerative colitis. Int J Mol Med. 2007;19:221-228.

10. Marlicz W, Zuba-Surma E, Kucia M, Blogowski W, Starzynska T, Ratajczak MZ. Various types of stem cells, including a population of very small embryonic-like stem cells, are mobilized into peripheral blood in patients with Crohn's disease. Inflamm Bowel Dis. 2012;18:1711-1722.

11. Boltin D, Kamenetsky Z, Perets TT, et al. Circulating bone marrow-derived CD45-/CD34+/CD133+/VEGF+ endothelial progenitor cells in adults with Crohn's disease. Dig Dis Sci. (Epub ahead of print). doi:10.1007/s10620-016-4234-y.

12. Wood JA, Colletti E, Mead LE, et al. Distinct contribution of human cord blood-derived endothelial colony forming cells to liver and gut in a fetal sheep model. Hepatology. 2012;56: 1086-1096.

13. Galiano RD, Tepper OM, Pelo CR, et al. Topical vascular endothelial growth factor accelerates diabetic wound healing through increased angiogenesis and by mobilizing and recruiting bone marrow-derived cells. Am J Pathol. 2004;164:1935-1947. 